\title{
Limit analysis of narrow support elements in W7-X considering the serration effect of the stress-strain relation at $4 \mathrm{~K}$
}

\author{
E. Briani ${ }^{\mathrm{a}}$, C. Gianini ${ }^{\mathrm{a}}$, F. Lucca ${ }^{\mathrm{a}}$, A. Marin ${ }^{\mathrm{a}}$, J. H. H. Fellinger ${ }^{\mathrm{b}}$, V. Bykov ${ }^{\mathrm{b}}$ \\ ${ }^{a}$ L.T.Calcoli SaS, Piazza Prinetti 26/B, 23807, Merate (LC), Italy \\ ${ }^{b}$ MPI fur Plasmaphysik (IPP) Wendelsteinstrabe I, D-17491 Greifswald, Germany
}

\begin{abstract}
The magnet support system of the Wendelstein 7-X (W7-X) fusion stellarator includes challenging components, called Narrow Support Elements (NSEs), placed between the Non Planar Coils (NPCs) at the inboard side and aimed at reducing deformation of the coils. NSEs are small contact elements, with special coating to reduce friction, that have to withstand high compressive and shear forces. The objective of this article is to demonstrate the structural reliability of the NSEs under electromagnetic loading (EML), taking into account in a conservative way the relevant material properties at cryogenic temperatures. To this purpose, an appropriate parametric local Finite Element (FE) model of one highly loaded NSE with its components (pad, pad frame and counter pad) and of a portion of the coils has been developed with ABAQUS code and isotropic elastic-plastic material model with hardening/softening has been used, in order to include the serration effect at $4 \mathrm{~K}$. Different mechanical limit analyses have been performed including consecutive steps of shrink fitting the NSEs in the coils, cooling down to $4 \mathrm{~K}$ and gradual increasing of the coil displacements induced by the EML.
\end{abstract}

Keywords: W7-X, magnet system, FE modeling, limit analysis, serration effect

\section{Introduction}

The magnet system of W7-X, the fusion stellarator presently under construction in Greifswald, Germany [1], includes 50 Non Planar Coils (NPCs) and 20 Planar Coils (PCs), which are divided in 5 identical modules along the toroidal direction ( $72^{\circ}$ each). Each module consists of two half-modules ( $36^{\circ}$ each) symmetric around the radial axis.

During the energization of the magnet system, the forces acting on the coils due to the electromagnetic loads (EML) deform the whole structure and the coils interact through some elements aiming at the limitation of the deformation of the coils. Among these components, some small contact elements, called Narrow Support Elements (NSEs), are located in the inboard side of two adjacent NPCs (see Fig.1): they have special coating to reduce friction and they have to withstand high compressive and shear forces.

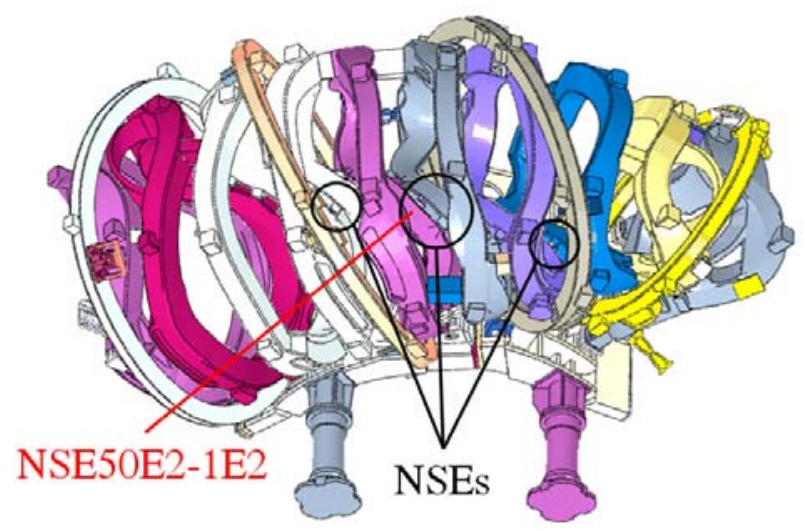

Fig.1 NSEs between adjacent NPCs

Two different types of NSEs have been designed (60 $\mathrm{mm}$ and $73 \mathrm{~mm}$ in diameter) to withstand up to 1
$\mathrm{MN}$ and 1.5 MN contact force respectively, up to $5 \mathrm{~mm}$ slipping and up to $1^{\circ}$ relative tilting angle [2 - 4].

Each NSE consists of a pad, a pad frame and a counter pad: the pad is an Al-Bronze element, fitting into the pad frame on the coil and it has a spherical surface that can get in contact with the counter surface on the other coil. The counter surface is either a separate stainless steel element or a machined surface of the coil case; its contact surface is perfectly planar to permit the sliding between the NPCs. The stainless steel pad frame fits into the coil with a small pin and it has the purpose to keep the pad in its correct orientation [2, 3]. Since the load distribution on NSEs is very sensitive to the initial (no energization of the coils) gap between the pad and the counter pad [2 - 4], the pre-tilting angle and the gap in the NSE have to be carefully designed and controlled.

In the past, two global models (GMs) of the $36^{\circ}$ submodule and $72^{\circ}$ module, elastic in material, have been developed with the ABAQUS code by L.T.Calcoli and IPP [4]: in these GMs also the NSEs have been included with a relatively coarse mesh to simulate the contact interaction between the NPCs.

The aim of the study presented here is to simulate the NSEs behavior during assembly, cool down and EM loading with consideration of the serration effect of the material at cryogenic temperature. Therefore a detailed and parametric non-linear local model was developed.

\section{Structure and analyses description}

\subsection{Finite Element Model}

The analyzed NSE is the NSE50E2-1E2, located between the two NPCs type 1 (see Fig.1) on the symmetry axis of the module. 
The developed local FE model consists of a refined mesh of each component of the NSE (see Fig.2): the pad (Ø $73 \mathrm{~mm}$ ), the pad frame (Ø $95 \mathrm{~mm})$ and the counter pad (Ø $95 \mathrm{~mm}$ ); moreover, a suitable portion of the coil cases is included to avoid local effects due to the boundary conditions in the interested area of the NSE. The Winding Pack (WP) enclosed into the coils is not included in the local model because of its relative flexibility.

Some parametric features are included in this model to modify the layout of the structure: so it is possible to adjust i) the pre-tilting angle of the pad and ii) its orientation with respect to the positioning pin, iii) the height of the pad frame bore and iv) the distance between the pad and the counter pad to change the relative position and rotation between them.

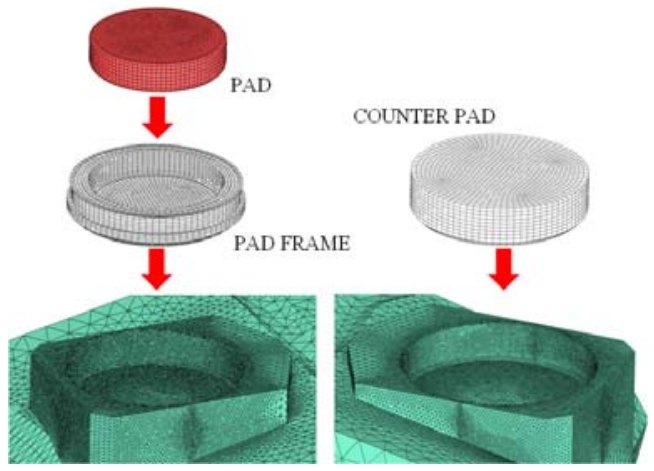

Fig.2 mesh of NSE components and their positioning into the coils

\subsection{Analyses procedure}

The analysis is divided into two phases: in phase 1 the procedure is tested and an understanding of the failure behavior is obtained. In phase 2 it is verified that the NSE can withstand the applied loads with sufficient safety margin.

In the phase 1 the structure is subjected to prescribed boundary conditions, including the simulation of the physical processes of 1) the shrink fit of the NSE components in the coils, 2) the cooling down from room temperature to operating cryogenic temperature and 3) the application of prescribed boundary conditions (see Section 2.3.1) until the failure of the structure. Failure is defined by either the $14 \%$ of the equivalent local plastic strain on the pad or the $3 \%$ of the average equivalent plastic strain on any critical cross section of the pad or the change of slope of the load-displacement curve of the whole structure.

In the phase 2 the local model has been subjected to the boundary conditions extracted from the ABAQUS $72^{\circ} \mathrm{GM}$ analysis which includes three steps: 1) preloading of the bolts and the dead weight of the structure, 2) cooling down to cryogenic temperature and 3) application of the forces due to the EML. During preload and cooling down, the shrink fitting and cooling down processes are simulated on the local model. To ensure that the required limit load on the NSE is reached, the EML in the GM is scaled by a sufficiently large factor (up to 3). For the analyzed NSE the required limit load is $1983 \mathrm{kN}$, which is 1.5 times the maximum normal force over all EML configurations.

Due to the strong non-linearity of the model, caused by the relevant materials properties, the gradual but continuous increasing in the contact interactions and the geometry discontinuities, the static implicit procedure did not converge to a full solution: therefore, the static response of the structure is simulated via an explicit quasi-static procedure.

\subsection{Initial and boundary conditions}

In both phases the shrink fit step is simulated applying an appropriate temperature to each NSE component to obtain the prescribed interference among the parts. In the cooling down step the temperature of the whole structure is brought from room temperature (293K) to the operating temperature of $4 \mathrm{~K}$.

The third step of the analyses is different for the two phases.

\subsubsection{Phase 1}

The initial $0^{\circ}$ pre-tilting angle and the $4 \mathrm{~mm}$ distance between pad and counter pad are imposed at the beginning of the analysis; with these initial conditions, the following boundary conditions, referred to a local Cartesian coordinate system (see Fig.3), are applied consecutively during the third step of the phase 1: a) 4 $\mathrm{mm}$ displacement in local $\mathrm{X}$ direction, b) $1^{\circ}$ tilting rotation around the local $\mathrm{Y}$ direction and $\mathrm{c}$ ) the gradually increasing of displacement in local $\mathrm{Z}$ direction until failure. Since the sequence of the application of the imposed boundary conditions could affect the structural response, a second analysis is performed applying all the boundary conditions simultaneously in one step. In this second analysis, the imposed displacement in local $\mathrm{Z}$ direction equals the displacement at failure of the first analysis. These two analyses are referred to as approach $\mathrm{A}$ and approach $\mathrm{B}$ respectively.

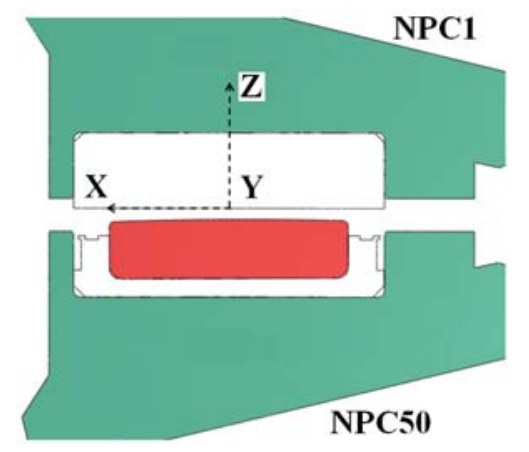

Fig.3 section of local FE model and local reference Cartesian coordinate system

\subsubsection{Phase 2}

An initial $0.3^{\circ}$ pre-tilting angle, at $75.2^{\circ}$ inclination with respect to the positioning pin and $4 \mathrm{~mm}$ gap between pad and counter pad are imposed at the beginning of the analysis; with these initial conditions, the sub-modeling automatic procedure of the ABAQUS code extracted the nodal displacements from the GM corresponding to the prescribed steps and applied them to the boundaries of the local model. 


\subsection{Material model}

The material properties are defined at room (293K) and at cryogenic temperatures (4K): the isotropic elasticplastic model with hardening/softening is used in order to include the serration effect. The serrated yielding, i.e. a zigzag shaped yield curve, is observed in tensile tests on many stainless steels at cryogenic temperatures. It is attributed to the strength loss induced by local heating of the plastic zone by conversion of plastic work into heat.

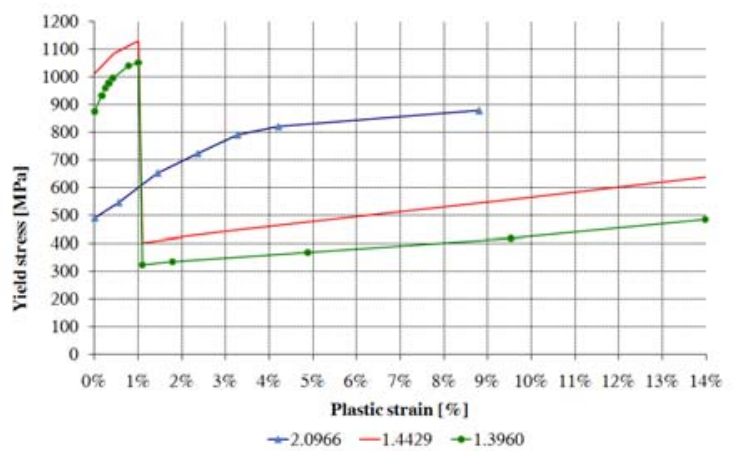

Fig.4 The materials stress-strain curve at $4 \mathrm{~K}$

To account for the serration effect, it is conservatively assumed that the yield stress of the stainless steels drops to the room temperature value upon $1 \%$ plastic strain. Fig. 4 shows the stress-strain curve at $4 \mathrm{~K}$ for the materials used: the Al-Bronze 2.0966 for pad, the stainless steel 1.4429 for the pad frame and the stainless steel 1.3960 for the coils. During the shrink fit and the cooling down steps, it is foreseen that no plastic deformations occur.

The friction coefficient among all surfaces is 0.3 , except between the pad and the counter pad where it is 0.1 in the phase 1 and 0.2 in the phase 2 .

Table 1: materials properties

\begin{tabular}{cccccc}
\hline Material & $\begin{array}{c}\text { Young Modulus } \\
{[\mathrm{GPa}]}\end{array}$ & $\begin{array}{c}\text { Poisson } \\
\text { coefficient }\end{array}$ & $\begin{array}{c}\text { Density } \\
{\left[\mathrm{kg} / \mathrm{m}^{3}\right]}\end{array}$ & $\begin{array}{c}\text { Thermal } \\
\text { expansion } \\
{[1 / \mathrm{K}]}\end{array}$ \\
\hline \multicolumn{7}{c}{$4 \mathrm{~K}$} & $\mathrm{RT}$ & & & \\
\hline 2.0966 & 122 & 118.8 & 0.28 & 7600 & $10.04 \mathrm{e}-6$ \\
1.4429 & 197.4 & 183.3 & 0.28 & 7800 & $9.7 \mathrm{e}-6$ \\
1.3960 & 158 & 151.7 & 0.3 & 7800 & $10 \mathrm{e}-6$ \\
\hline
\end{tabular}

\section{Results}

\subsection{Phase 1}

The results of the phase 1 show that the failure of the structure is due to yielding of the coils, shown by a steep drop in the load-displacement curve (see Fig. 5): the compression load applied to the NSE causes a "bending" deformation of the NPC and an increase of the plastic strain across the middle sections of the coil. It is affected by the part of the coil case included in the model and the exclusion of the WP. In addition, the bore in the coils deforms with full yielding of the thin lateral shoulders. The two approaches produce similar results.
The maximum value reached in the compressive force is about $3.24 \mathrm{MN}$ at a total displacement of 8.71 $\mathrm{mm}$ in local Z direction (see Fig.5, approach A). Fig.6 shows the contour plots of the Von Mises stress and of the equivalent plastic strain (PEEQ) on two orthogonal middle sections of the NSE at failure in approach A. During the analyses the serration effect is clearly visible on the coils and the pad frame (see Fig.7).

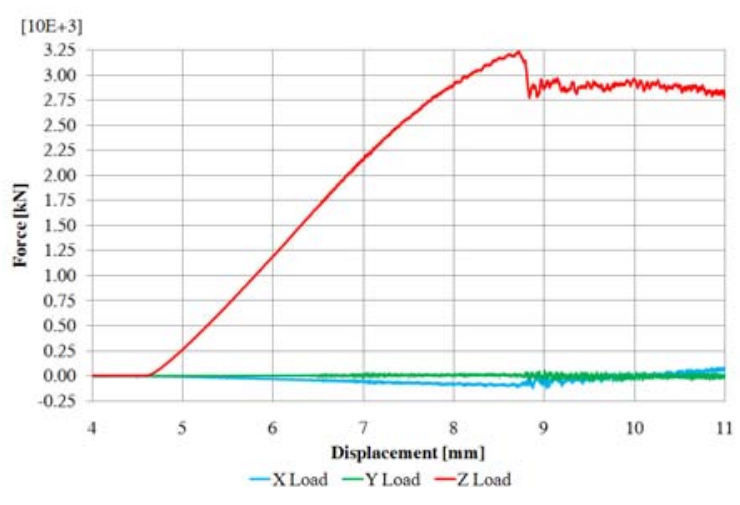

Fig.5 Load-displacement curve in approach A

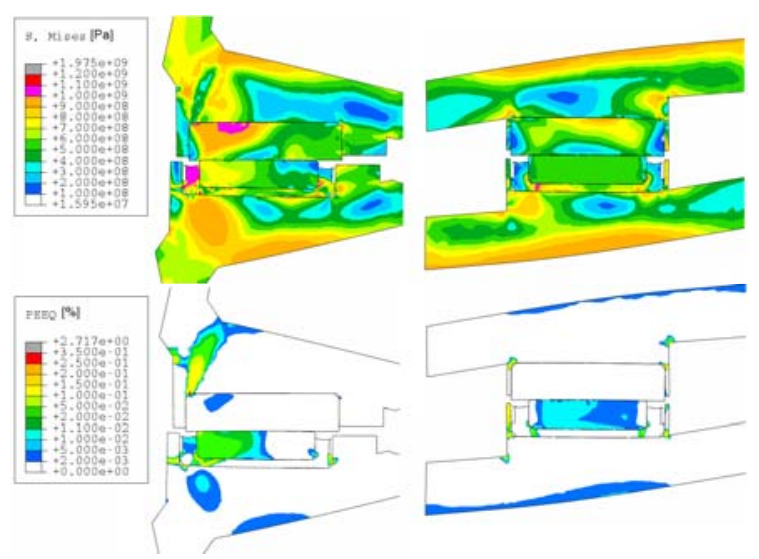

Fig.6 The Von Mises stress and the PEEQ contour plots on the middle sections at failure in approach $\mathrm{A}$
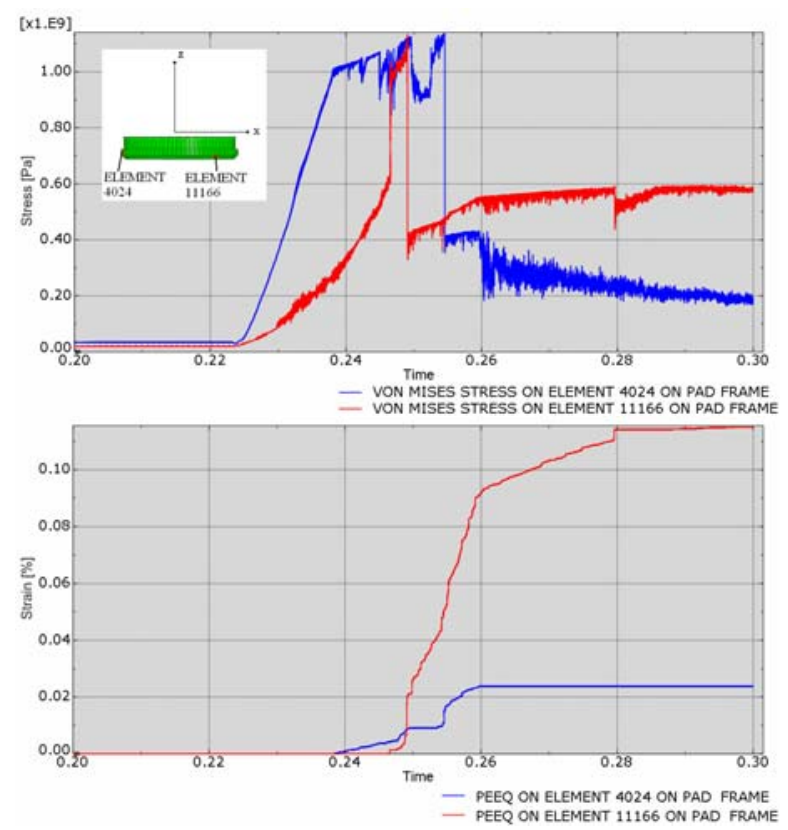

Fig.7 Von Mises stress and PEEQ vs. time on marked points of pad frame 


\subsection{Phase 2}

The boundary conditions extracted from the GM are applied on the edges of the sub-model, causing local plasticization and stress concentrations that, however, do not affect the behavior of the NSE. As expected, the submodel structure is less stiff than the GM: in fact, it reaches the required limit load for an EM load factor higher (about 1.95) than that of the GM structure (about 1.7). Moreover, the shear force in the sub-model follows the same trend as the GM, although with higher magnitude. Notably, the shear force remains in both cases far below the frictional limit of 20\%. Fig.8 demonstrates the normal and shear contact forces acting on the NSE in the GM and sub-model.
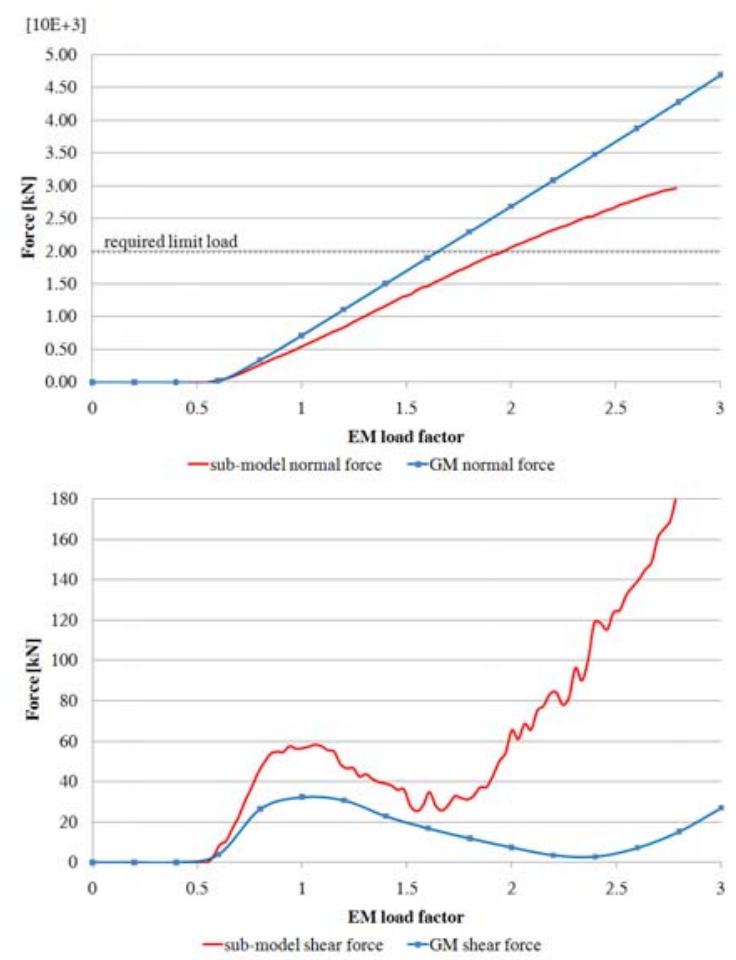

Fig.8 The normal and shear forces on the NSE in the GM and sub-model

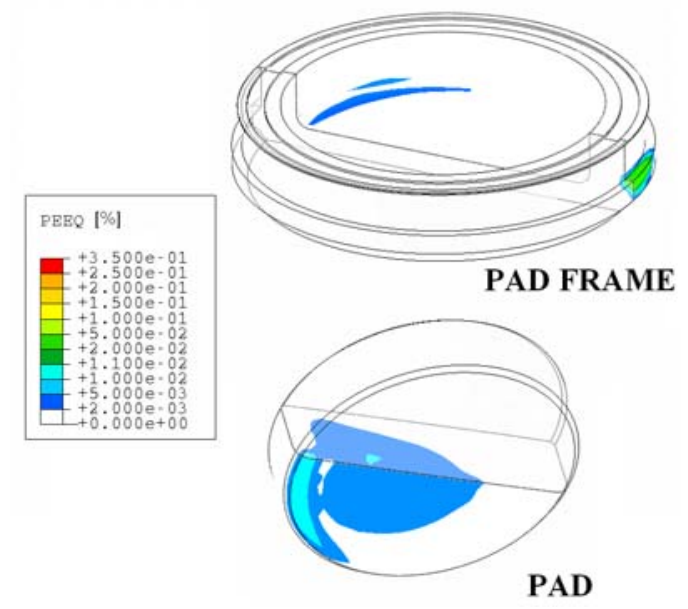

Fig.9 The PEEQ contour plots on half portion of the NSE components at about 1.95 EM load factor

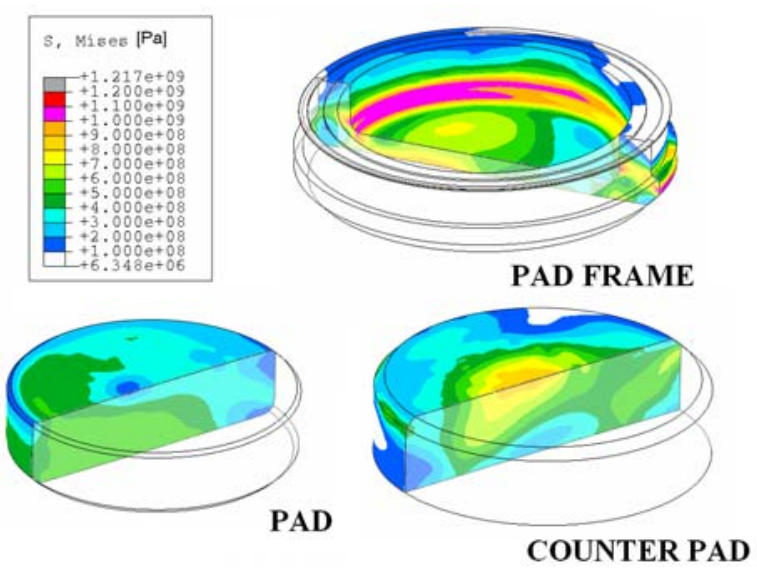

Fig.10 The Von Mises stress contour plots on half portion of the NSE components at about 1.95 EM load factor

The maximum PEEQ reached at about 1.95 EM load factor is $0.94 \%$ on the pad, $4.5 \%$ on the pad frame and less than $0.1 \%$ on the counter pad (see Fig.9 and Fig.10). The serration effect leading to localization of plastic strain is visible on the lateral shoulder of the pad frame.

\section{Conclusion}

The NSEs are small sensitive components that have to withstand high compressive and shear forces: the FE method permits to reproduce and evaluate in a suitable manner their behavior during the processes of assembly, start up and loading of the magnetic system structure, considering the relevant material properties at cryogenic temperature including the serration effect.

The developed parametric local model permits to evaluate the effect of different initial configurations at several EMLs, with a manageable and quickly adaptable method.

The performed analyses (phase 1) have shown that the complete failure of the structure is caused by the folding up of the coils case under the increasing compression displacement; the critical EM load condition analyzed in the phase 2 demonstrated that the required limit load can be resisted, without compromising the mechanical behavior of the NSE.

The presented activity will proceed with the development of the parametric sub-models of three NSEs selected on the basis of the high normal force during the operation and their high tilting angles. The procedure followed in the phase 2 for the first NSE will be performed also on these other NSEs.

\section{References}

[1] L. Wegener, Fusion Engineering and Design 84, 106-112 (2009)

[2] B. Heinemann, M. Gasparotto, C. Damiani, et al., IEEE 2005, ISBN: 0-4244-0150-X

[3] C. Damiani, S. Baumel, A. Benndorf, V. Bykov, A. Cardella, et al., IEEE 2007, ISBN: 978-1-4244-1194-8

[4] V. Bykov et al., Fusion Engineering and Design 84, 215219 (2009) 